\title{
SURVEY OF RECENT RESULTS ON ELECTRON CLOUD EFFECTS IN PHOTON MACHINES*
}

\author{
K. Harkay ${ }^{\dagger}$ and R. Rosenberg ${ }^{\dagger}$, Advanced Photon Source, \\ Argonne National Laboratory, Argonne, IL 60439, USA \\ Z.Y. Guo ${ }^{\ddagger}$ and Q. Qin ${ }^{\ddagger}$, Institute of High Energy Physics, \\ Chinese Academy of Sciences, Beijing, 100039, P.R. China
}

\begin{abstract}
Electron cloud interactions with high-energy beams are believed responsible for various undesirable effects ranging from vacuum degradation to collective beam instabilities. An important source of uncertainty in predicting electron cloud effects for a given machine lies in understanding in detail the processes relating to the generation of the cloud. The Advanced Photon Source (APS) has taken a lead role in the development of techniques for and the interpretation of direct measurements of the electron cloud distribution. Through such data, good progress has been made in placing realistic limits on critical input parameters in computer models relating to cloud production for actual accelerator chamber surfaces and geometries. Specially constructed electron detectors, using designs based on those first implemented at the APS storage ring, have been installed or are planned at a number of electron, positron, and proton rings to directly measure the properties of the electron cloud. Highlights will be presented of recent results from the APS, and a comparison is made between measurements at the APS and recent results at the Beijing Electron Positron Collider. These results illuminate machine conditions in which secondary processes become important.
\end{abstract}

\section{INTRODUCTION}

Numerous observations have been reported in photon machines - dedicated synchrotron light sources and highenergy electron-positron colliders - of degradation of machine performance due to electron cloud effects [1][2] [3][4][5]. A summary of the mechanisms responsible for electron cloud generation, including discussions of photoelectron and secondary electron processes and of cloud-beam interaction, can be found in Refs. [6][7].

In order to directly characterize the electron cloud distribution experimentally, a planar, retarding-field electron energy analyzer (RFA) was designed and implemented at the Advanced Photon Source (APS) [8][9]. RFA-type detectors have been installed or are planned at a number of rings: Proton Storage Ring (Los Alamos National Laboratory) [10]; Beijing Electron

\footnotetext{
* Work supported by the U.S. Department of Energy, Office of Basic Energy Sciences under Contract No. W-31-109-ENG-38.

** Work supported by the Chinese National Foundation of Natural

Sciences, Contracts 19875063-A050501 and 19975056-A050501.

†harkay@aps.anl.gov and rar@aps.anl.gov

guozy@mail.ihep.ac.cn and qinq@mail.ihep.ac.cn
}

Positron Collider (BEPC) (Institute of High Energy Physics (IHEP), P.R. China) [11]; KEK B-factory (High Energy Accelerator Research Organization (KEK), Japan); Alternating Gradient Synchrotron (AGS) Booster (Brookhaven National Laboratory); PEP-II B-factory (Stanford Linear Accelerator Center); and Super Proton Synchrotron (SPS) (CERN).

Under specific machine conditions at the APS and using the RFA detectors, evidence of beam-induced multipacting (BIM) was observed for bunch currents above $1-$ $1.5 \mathrm{~mA}$. However, no clear evidence of cloud-induced collective effects were seen. In contrast, experiments carried out at the BEPC show evidence of the photoelectron instability (PEI) [5]. Simulations using a physical model of PEI give qualitative agreement with the observations.

\section{COMPARISON OF APS AND BEPC}

When electron detectors based on the ANL RFA design were installed at the BEPC in 2000, an opportunity arose to study the properties of the electron cloud in a machine where the PEI is observed, and to compare the results with the APS. Due to the absence of an antechamber, photoelectron emission is expected to dominate at the BEPC, while at the APS, secondary electron emission is expected to dominate only under BIM resonance conditions. With the exception of Sect. 2.3, all the comparison data were acquired with positron beams.

The relevant machine parameters for the two rings are listed in Table 1. Although the beam energies and ring sizes are rather different, the chamber dimensions are comparable. The beam-cloud dynamics depends primarily on the bunch charge and spacing, while the photo- and

Table 1: Machine Parameters for APS and BEPC

\begin{tabular}{|l|c|c|c|}
\hline & & APS & BEPC \\
\hline Beam energy & $\mathrm{GeV}$ & 7 & 1.3 \\
\hline Circumference & $\mathrm{m}$ & 1104 & 240.4 \\
\hline RF frequency & $\mathrm{MHz}$ & 351.9 & 199.5 \\
\hline Minimum bunch spacing & $\mathrm{ns}$ & 2.8 & 5.0 \\
\hline Harmonic number & - & 1296 & 160 \\
\hline Chamber semi-axes $(a, b)$ & $\mathrm{mm}$ & $42.5,21$ & 60,29 \\
\hline Antechamber height & $\mathrm{mm}$ & 10 & none \\
\hline Chamber material & - & $\mathrm{Al}$ & $\mathrm{Al}$ \\
\hline Distance from dipole & $\mathrm{m}$ & 9.25 & $0.23(\mathrm{e}+)$ \\
magnet end to RFA & & $(\mathrm{e}+/ \mathrm{e}-)$ & $1.47(\mathrm{e}-)$ \\
\hline Dipole bend angle & $\mathrm{rad}$ & 0.07854 & 0.1547 \\
\hline Dipole length & $\mathrm{m}$ & 3.06 & 1.597 \\
\hline Bunch length $(\mathrm{rms})$ & $\mathrm{cm}$ & 1 & 3 \\
\hline
\end{tabular}


secondary emission processes are influenced in large part by the chamber surface properties and geometry. The vacuum chambers in both machines are made of aluminum, whose surface oxide has one of the highest secondary electron yield coefficients of materials typically used.

In the experiments, the electron cloud is measured at a fixed location in the ring. The beam energy, bending magnet radiation opening angle, and distance to the detector determine the average photon flux on the chamber wall at the location of the detector. The presence or absence of an antechamber determines what fraction of these photons above an energy threshold (typically $\sim 4 \mathrm{eV}$ ) contribute to photoproduction.

The cross-sections of the APS and BEPC chambers are shown in Fig. 1. The locations of the RFAs are also shown schematically to scale. In a detailed comparison of the two cases, several effects should be considered. At BEPC, the diameter of the RFA aperture is about two times larger than at the APS. At the APS, the aperture is further reduced by a factor of $2-3$ (to about $1 \mathrm{~cm}^{2}$ ) because the detector is mounted behind a vacuum penetration for $\mathrm{rf}$ shielding. The orientations of the detectors are different, and would need to be accounted for. Also, the detector at BEPC was installed adjacent to a dipole magnet in a region of non-zero fringe field (the detector itself was magnetically shielded). The detector at the APS is in a field-free region, and the stray field from a nearby ion pump was eliminated. For the purposes of this paper, these effects are discussed only qualitatively in the presentation of the experimental results.
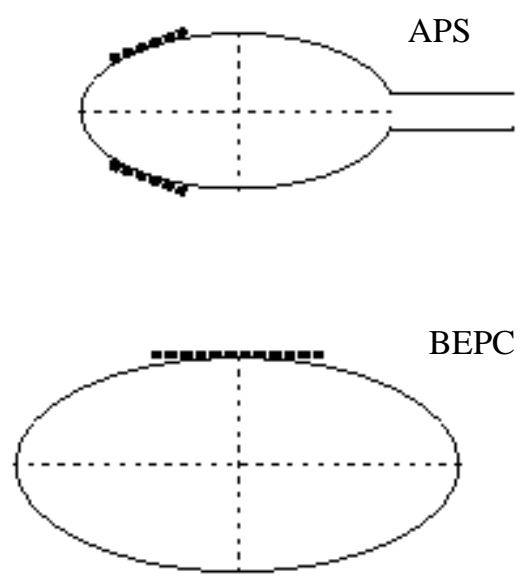

Figure 1: Schematic showing mountings of detectors on APS and on BEPC vacuum chambers (half-scale).

\subsection{Bunch Spacing}

The electron detector signals are compared as a function of positron bunch spacing and bunch current in Fig. 2. A dramatic amplification of the cloud is observed at the APS at a 20-ns spacing (7 rf buckets) [9]. A related pressure rise is also observed, indicative of secondary electron-induced gas desorption, and giving independent evidence [12] of beam-induced multipacting (BIM). To look for possible BIM effects at BEPC, we scanned over a range of bunch currents and spacings near the expected resonant condition for BIM. In the impulse approximation, the momentum kick by the beam of an electron at a radial position, $r$, from the beam is $\Delta p_{e}=2 m_{e} c r_{e} N_{e} / r$, where $r_{e}=2.8 \times 10^{13} \mathrm{~cm}$ is the classical electron radius and $N_{e}$ is the number of beam particles. The resonant condition involves the electron drift time across the chamber between bunch passages and the momentum kick producing the highest yield of secondary electrons. By analogy with the APS ( $20 \mathrm{~ns}$ bunch spacing and $3 \times 10^{10}$ positrons), we expect to observe BIM at BEPC with 30-ns spacing with the same bunch population. The data in Fig. 2 do not appear to show any amplification effects at BEPC (the data up to 30-ns bunch spacing were acquired with a different lattice than that above 40-ns spacing).

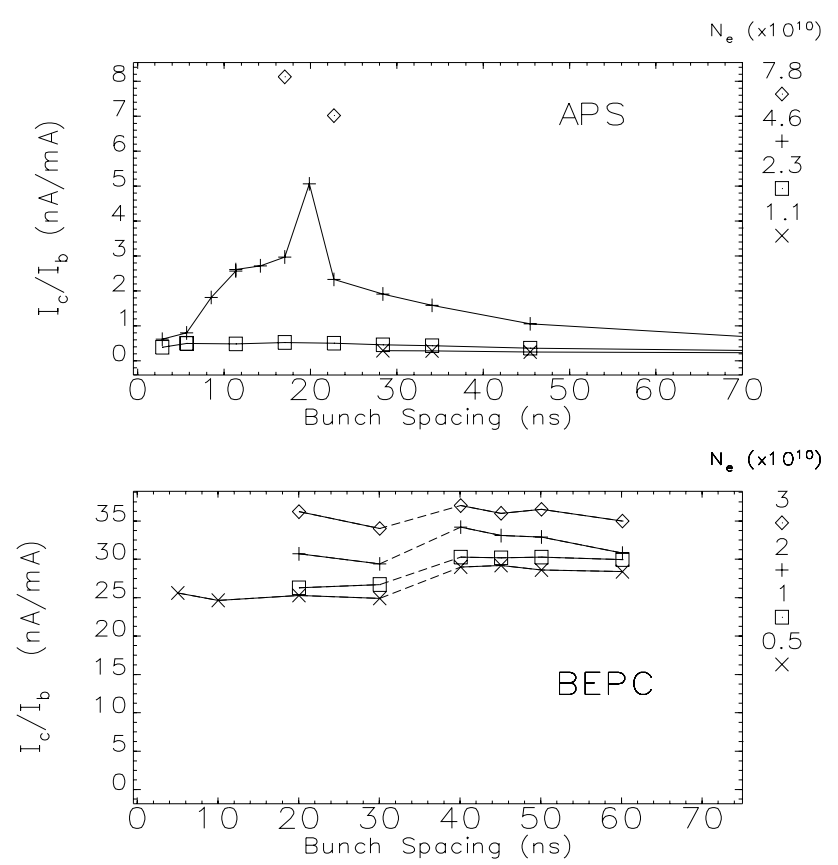

Figure 2: Electron detector current $\left(\mathrm{I}_{\mathrm{c}}\right)$ vs. bunch spacing as a function of positrons/bunch $\left(\mathrm{N}_{\mathrm{e}}\right)$, normalized to total beam current ( $\left.\mathrm{I}_{\mathrm{b}}\right)$ (APS: 10 bunches; BEPC: 5 bunches).

\subsection{Bunch Train}

The characteristics of the electron cloud buildup over positron bunch trains of varying length $\left(\mathrm{N}_{\mathrm{b}}\right)$ are compared as a function of bunch current in Fig. 3. In the APS, the buildup was most pronounced at the 20-ns spacing, as expected. The buildup rate is bunch-current-related, and for $2 \mathrm{~mA} / \mathrm{bunch}$, the normalized detector signal reaches saturation (linear growth) after 30 bunches $(0.6 \mathrm{~ms})$. A pressure rise of a factor of 20 was observed for 50 bunches over the pressure without BIM (nominally 0.5 nTorr at $100 \mathrm{~mA}$ ) [9]. The electron cloud buildup at BEPC, however, appears to be largely saturated over the full range of $\mathrm{N}_{\mathrm{b}}$ values. The case of $2 \mathrm{~mA} / \mathrm{bunch}$, however, does show a small amount of nonlinearity. The beam was stable in all cases. The slope of $I_{c} / I_{b}$ is nearly identical for a single-bunch $(27 \mathrm{nA} / \mathrm{mA})$ as for multibunch. Vacuum pressure data were not recorded. 

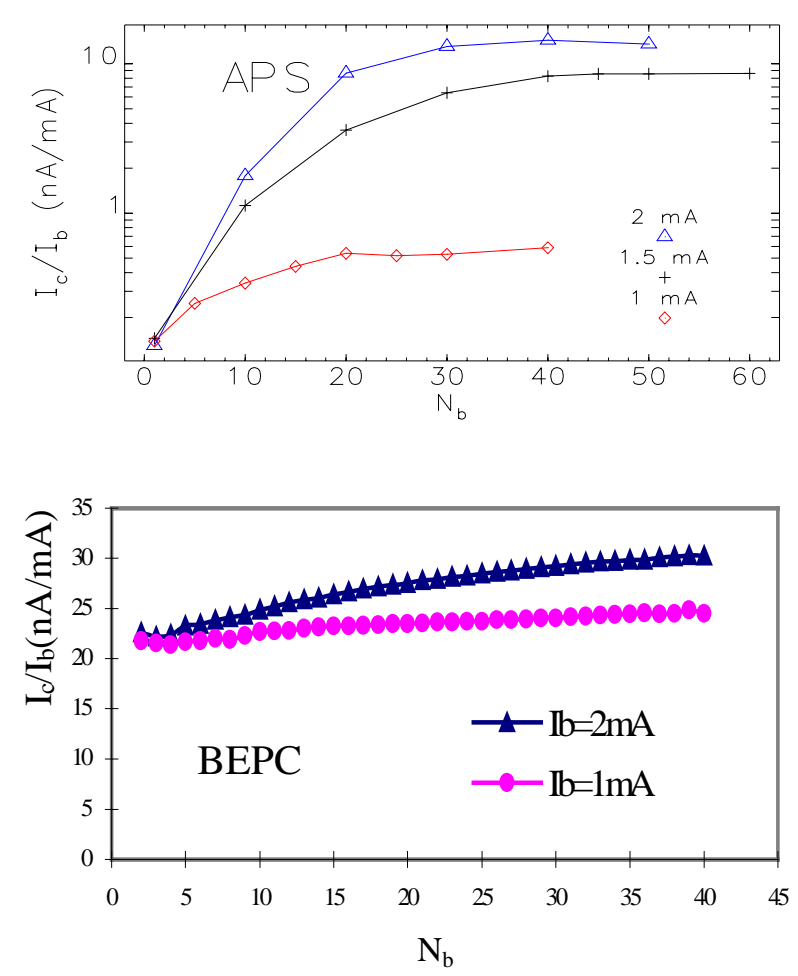

Figure 3: Electron detector current (normalized to total beam current) vs. increasing bunch number $\left(\mathrm{N}_{\mathrm{b}}\right)$ as a function of bunch current (bunch spacing for APS is $20 \mathrm{~ns}$, and for BEPC is $10 \mathrm{~ns}$ ). Note that the APS data is plotted on a $\log$ scale.

\subsection{Positron vs Electron Beam}

At BEPC, measurements were made with electron and positron beams stored separately, but during the same study period. The scan of detector current vs. retarding voltage is shown in Fig. 4, and shows that the positron signal is about six times higher than the electron signal. It should be noted that the detector is positioned six times closer to the upstream dipole radiation source for the positron beam compared to the electron beam (see Table 1). In addition, we expect that the deflection of electron cloud electrons into the detector for the two species will yield different collection rates. The data for the APS show a similar detector current ratio for positron and electron beams, with bunches at the minimum spacing. However, it should be noted that at the APS, the data were acquired on two separate occasions: before and after a long shutdown during which the magnet polarities were reversed to change from positron to electron operation. The data shown in Fig. 4 were thus acquired with different surface conditioning (integrated current with positrons: $62 \mathrm{Ah}$; electrons: $99 \mathrm{Ah}$ ), but otherwise the same machine conditions. The detector current ratio between positrons and electrons is furthermore a function of the bunch spacing, giving a ratio of only about 2 for bunches spaced far apart (360 ns).
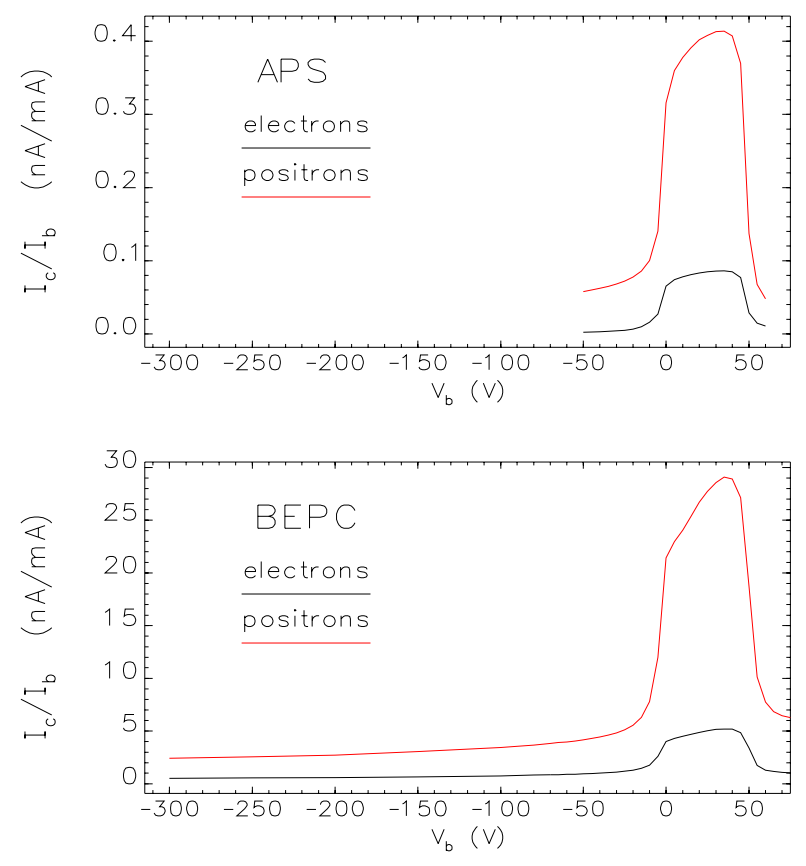

Figure 4: Comparison of detector signals for positron and electron beams (APS: 10 bunches, $20 \mathrm{~mA}, 2.84$-ns spacing; BEPC: 160 bunches, $10 \mathrm{~mA}, 5$-ns spacing). BEPC single-bunch results are almost identical to the multibunch case shown.

\subsection{Electron Cloud Effects}

As described above, the most notable electron cloud effect observed at the APS is a pressure rise resulting from beam-induced multipacting. The anomalous pressure rise is observed above a threshold of between 1 and 1.5 $\mathrm{mA} /$ bunch at the resonant bunch spacing $(20 \mathrm{~ns})$. At BEPC, what is believed to be a PEI is observed for uniform fills of every bucket or every other bucket (160 and 80 bunches, respectively) with positron beams. The instability intensity threshold with 160 bunches is about 10 $\mathrm{mA}$. The electron detector current was recorded below and above the stability threshold, shown in Fig. 5. The electron cloud increases linearly with beam current. This appears to indicate that the beam does not perturb the cloud above the instability threshold, although the reverse is expected if the instability is a PEI.

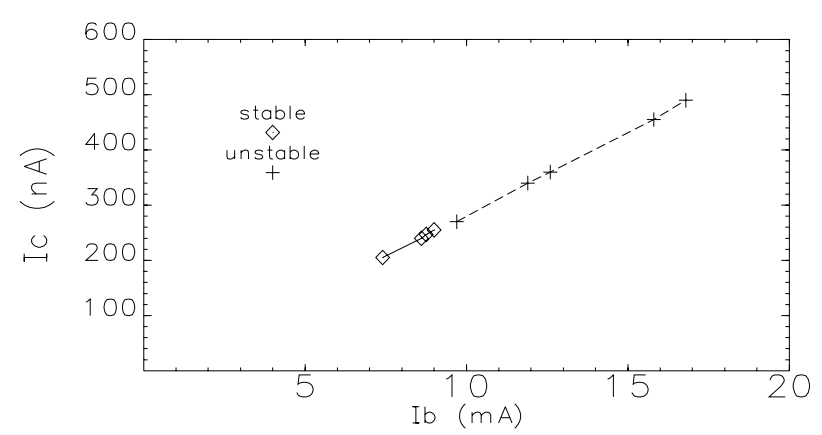

Figure 5: Electron detector signal below and above instability threshold with full, 160-bunch fill at BEPC. 


\section{RECENT APS RESULTS}

\subsection{Surface Conditioning}

Much work has been done to quantify surface conditioning effects in the laboratory for accelerator materials; notably, at CERN [7] and at SLAC [7][13]. To avoid or minimize electron cloud effects, of interest are techniques to reduce the secondary emission using a combination of surface preparation and electron bombardment. Good progress has been made in this area, based on bench data.

As expected, RFA measurements at the APS show the results of surface conditioning: the electron flux at the detectors decrease as a function of the integrated beam current, usually measured in Amp-hours (Ah). The amount by which electron detector signal levels were reduced from the time the new chamber and detectors were installed depended also on the temporal distribution of the bunches; i.e., whether BIM was induced. After 80-100 Ah of operation, the electron cloud signals were reduced by $20 \%$ for non-BIM (large bunch spacing) and by $50 \%$ at the resonant, 20-ns spacing.

In bench measurements, the conditioning is usually quantified in terms of the electron dose on the surface. Conversion of the APS integrated current to electron bombardment rate would require detailed knowledge of the history of operation. Assuming standard 100-mA operation, we can estimate the average bombardment rate using RFA measured data. For $100 \mathrm{Ah}$ at standard operation, we obtain a dose of about $4 \times 10^{17}$ electrons $/ \mathrm{cm}^{2}$ (the detector transmission efficiency is not taken into account).

\subsection{New Detector Design}

For the purposes of measuring the electron cloud flux at the chamber surface, the RFA shows clear advantages over a simple, unshielded collection plate. The collection length changes as the plate is biased, and secondary emission from the surface - itself a function of incident electron energy - affects the true signal. The RFA is an integrating device with reasonably good energy resolution for perpendicular incidence, but suffers from reduced resolution for angular incidence. Ongoing simulation studies of the APS [14] indicate how important a good model of the energy distribution is in predicting electron cloud effects; this motivated a study of alternate electron detector designs at the APS.

The Bessel Box Analyzer (BBA) is perhaps the simplest energy-dispersive analyzer. It is sometimes referred to as a "pill box" and is shown schematically in Fig. 6 [15]. The BBA consists of entrance and exit apertures held at potential $\mathrm{V}_{\mathrm{a}}$, a body with potential $\mathrm{V}_{\mathrm{b}}$, and a collector. At the center of the body is a circular "stop" whose diameter is slightly larger than the entrance aperture, which prevents direct, on-axis electrons from being transmitted through the exit aperture. It is also maintained at $V_{b}$. The difference between $\mathrm{V}_{\mathrm{b}}$ and $\mathrm{V}_{\mathrm{a}}, \Delta \mathrm{V}$, determines the pass energy of the analyzer. A comparison of the BBA and RFA bench measurements and measurements with beam are described in Ref. [15].

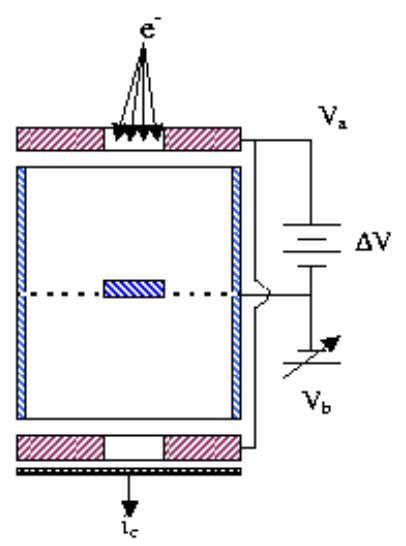

Figure 6: Schematic diagram of the Bessel Box Analyzer. The difference potential $\Delta \mathrm{V}=\left(\mathrm{V}_{\mathrm{a}}-\mathrm{V}_{\mathrm{b}}\right)$ determines the pass energy of the detector.

The RFA is simple to construct and has large transmission, but analysis of the energy spectra is complicated. While the BBA has relatively poor transmission, it offers the potential of direct analysis of the energy spectrum. Preliminary results from the BBA installed in the APS storage ring are intriguing. In the future we will devote more time to experiments and analysis using the BBA and also hope to incorporate an electron multiplier for signal amplification, which should allow us to perform timeresolved measurements.

Challenges remain in the detection of the very lowenergy electrons in the cloud. The secondary electron yield of electrons with $<1 \mathrm{eV}$ incident energy plays an important role in the electron cloud generation, but is not very well understood. Also of interest would be a measure of the electron cloud distribution in the dipoles. The signals in a device such as the RFA are affected by unshielded magnetic fields, and the results are therefore difficult to interpret. This problem was demonstrated dramatically in the BEPC studies before the RFA was shielded from the fringe fields from the adjacent bending magnet [11].

\section{ACKNOWLEGMENTS}

The authors gratefully thank M. Furman and S. Heifets for enlightening discussions and suggestions, the BEPC team, the hospitality of IHEP during a recent visit by the first author, and the collaboration to study the PSR instability.

\section{REFERENCES}

[1] M. Izawa, Y. Sato, T. Toyomasu, Phys. Rev. Lett. 74, 5044 (1995).

[2] K. Ohmi, Phys. Rev. Lett. 75, 1526 (1995); Proc. of 1997 PAC, 1667 (1998); and KEK Report 97-7 or IHEP/BEPC/AP/97-09.

[3] G. Rumolo, F. Zimmermann, H. Fukuma, and K. Ohmi, "Electron Cloud Studies for KEKB," these proceedings. 
[4] J. Seeman and A. Kulikov, "The Electron Cloud Instability at PEP-II," these proceedings.

[5] Z.Y. Guo et al., Proc. of 1997 PAC, 1566 (1998), Proc. of 1998 EPAC, 957 (1998), Proc. of 1998 APAC, 432 (1998).

[6] M. Furman, Proc. of Workshop on Multibunch Instabilities (MBI97), KEK Proc. 97-17, 170 and 234 (1997) and Proc. of 1997 PAC, 1617 (1998).

[7] For a summary, see Proc. of ICFA Workshop on Two-Stream Instabilities, Santa Fe, NM, Feb 16-18, 2000, http://www.aps.anl.gov/conferences/icfa/twostream.html.

[8] R.A. Rosenberg and K.C. Harkay, Nucl. Instrum. Meth. A 453, 507 (2000).

[9] K.C. Harkay and R.A. Rosenberg, Proc. of 1999 PAC, 1641 (1999).
[10] R.J. Macek et al., "Electron Proton Two-Stream Instability at the PSR," these proceedings.

[11] Z.Y. Guo et al., "Experiment to Measure the Electron Cloud at BEPC," these proceedings.

[12] O. Grobner, Proc. of the $10^{\text {th }}$ Int'l Conf. on High Energy Accel., Protvino, 277 (1977).

[13] R. Kirby and F. King, "Secondary electron emission yields from PEP-II accelerator materials," Report No. SLAC-PUB-8212, submitted to NIM-A.

[14] M.A. Furman et al., "Electron-Cloud Measurements and Simulations for the APS," these proceedings.

[15] R.A. Rosenberg and K.C. Harkay, "Design and Implementation of Simple Electron Detectors for Accelerator Diagnostics," these proceedings. 